Supporting Information

\title{
Controlled Engineering of Nano-Povidones for Efficient Iodine Recovery and Antibacterial Reutilization
}

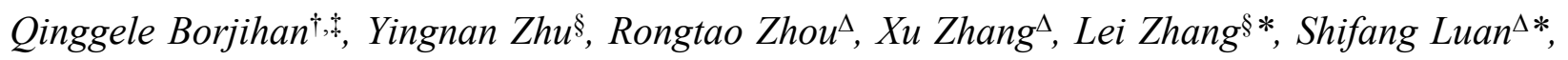
Alideertu Dong ${ }^{\dagger,+*}$

†College of Chemistry and Chemical Engineering, Inner Mongolia University, Hohhot 010021, People's Republic of China

Engineering Research Center of Dairy Quality and Safety Control Technology, Ministry of Education, Inner Mongolia University, Hohhot 010021, People's Republic of China

§Department of Biochemical Engineering, School of Chemical Engineering and Technology, Frontier Science Center for Synthetic Biology and Key Laboratory of Systems Bioengineering (MOE), Key Laboratory of Systems Bioengineering (Ministry of Education), and Collaborative Innovation Center of Chemical Science and Engineering (Tianjin), Tianjin University, Tianjin 300072, People's Republic of China

${ }^{\Delta}$ State Key Laboratory of Polymer Physics and Chemistry, Changchun Institute of Applied Chemistry, Chinese Academy of Sciences, Changchun 130022, People's Republic of China.

*lei_zhang@tju.edu.cn (L.Z.); sfluan@ciac.ac.cn (S.L.); dongali@imu.edu.cn (A.D.)

Number of pages: 12

Number of tables: 0

Number of figures: 10 


\section{Table of Contents}

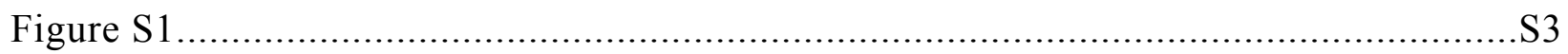

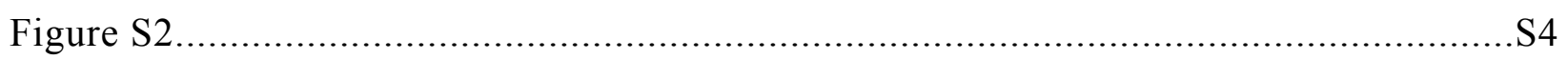

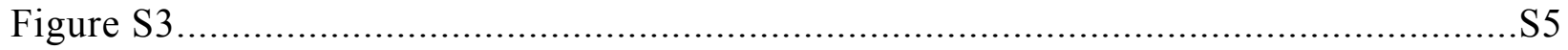

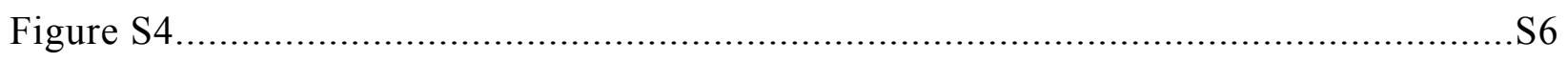

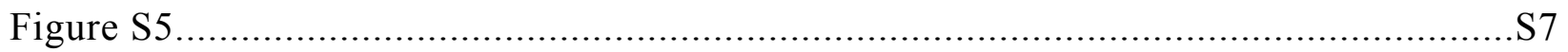

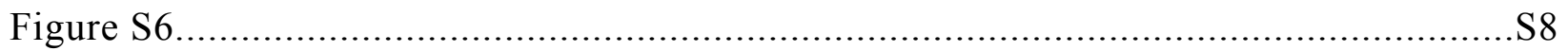

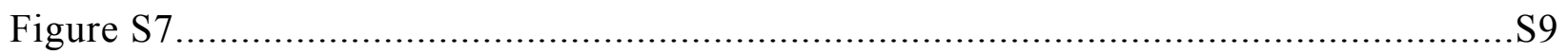

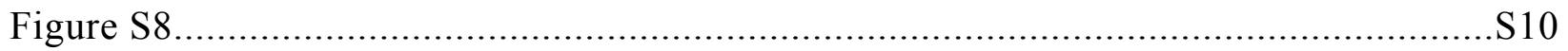

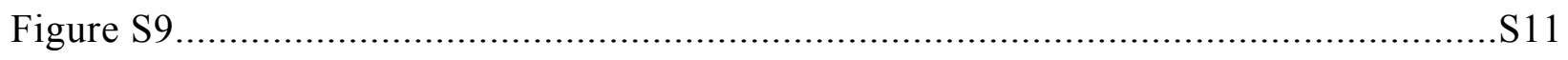

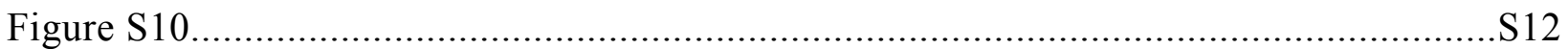


A

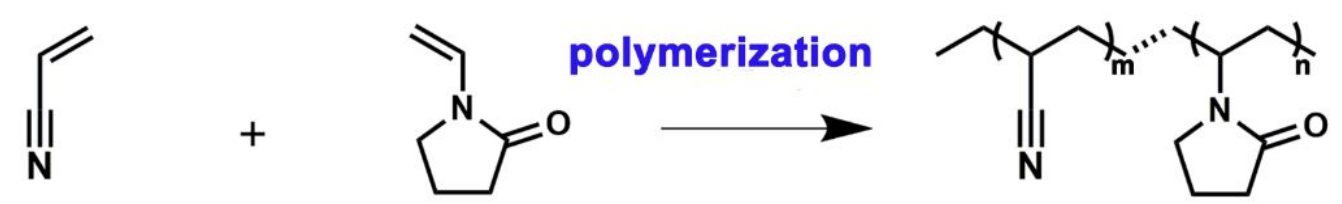

B<smiles>CCC(C#N)CCCCC(CC)N1CCCC1=O</smiles>

Figure S1. Schematic representation of the synthetic process of (A) P(AN-NVP) and (B) P(ANNVP)-I. 

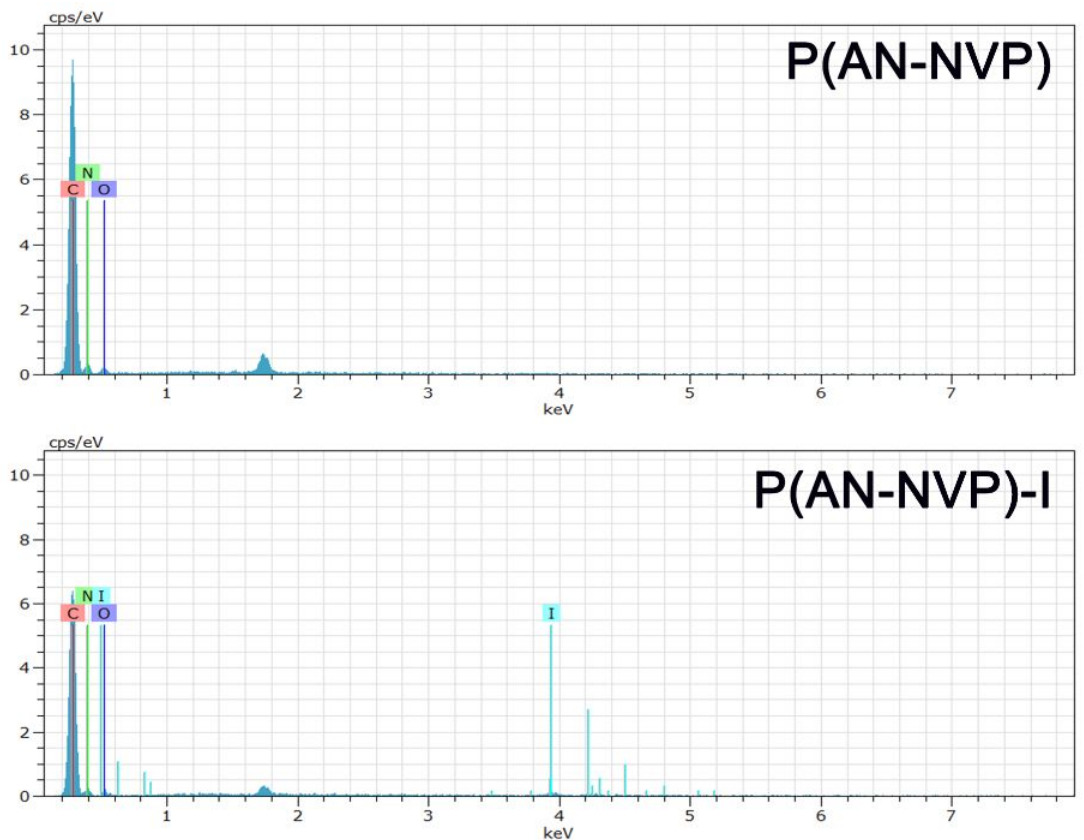

Figure S2. EDX spectrum of $\mathrm{P}(\mathrm{AN}-\mathrm{NVP})$ and $\mathrm{P}(\mathrm{AN}-\mathrm{NVP})-\mathrm{I}$. 


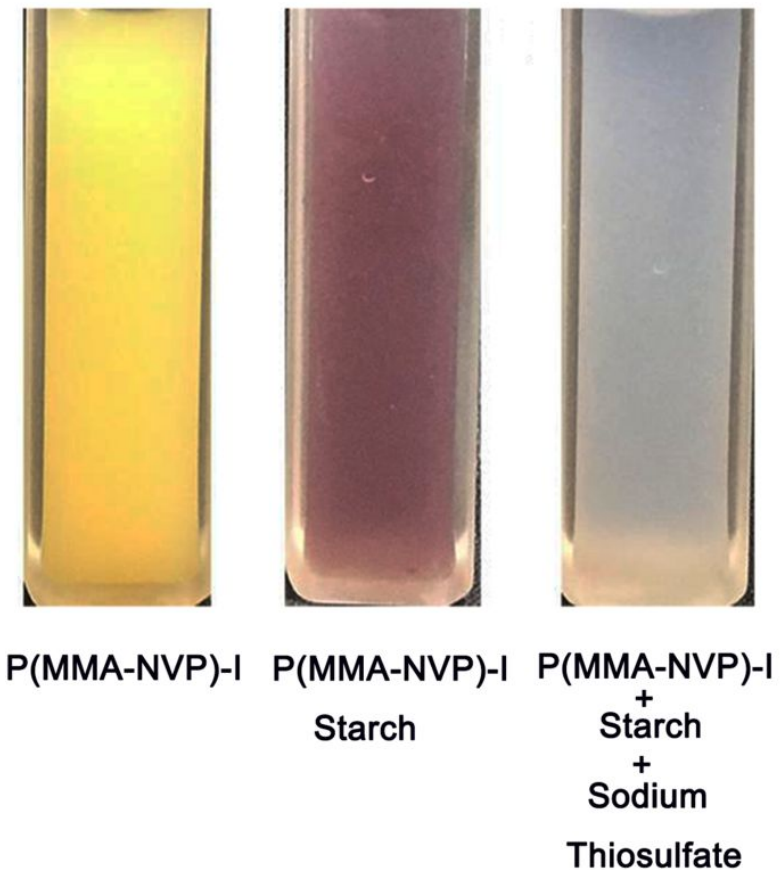

Figure S3. Photographs showing color changes in the P(MMA-NVP)-I suspension involved in the iodometric reaction. 


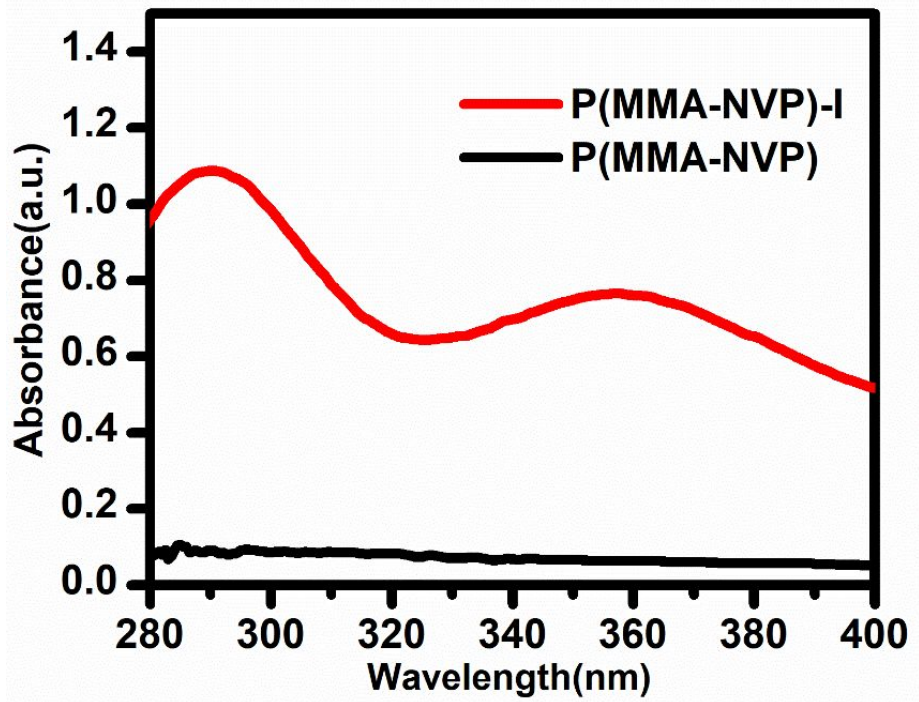

Figure S4. UV-vis spectra of P(MMA-NVP) and P(MMA-NVP)-I. 


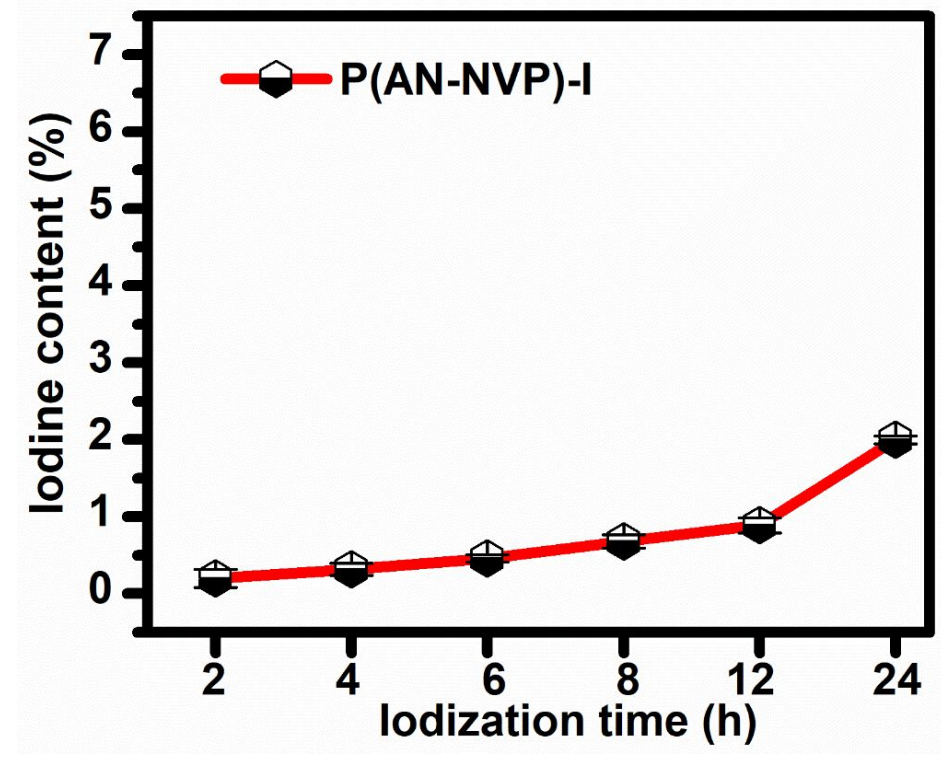

Figure S5. Iodine content of P(MMA-NVP)-I as a function of iodization time. 


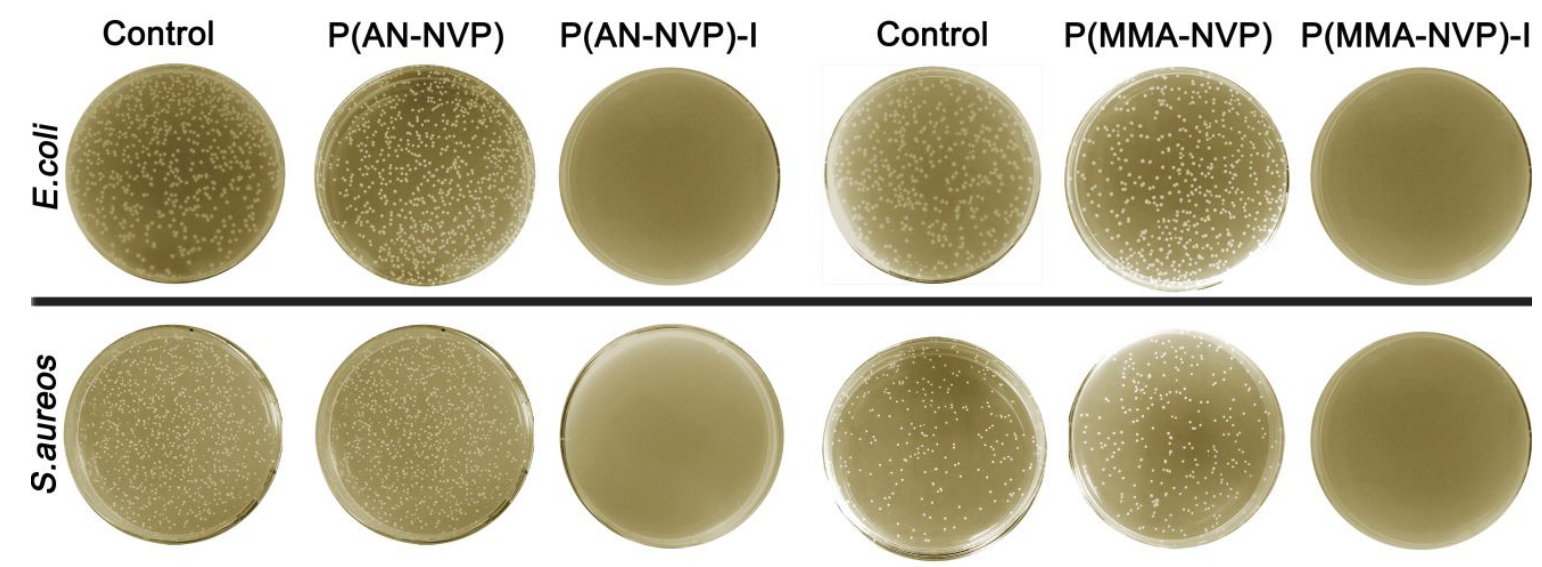

Figure S6. Photographs of the bacterial culture plates of E. coli and $S$. aureus after treatment respectively with $0.5 \mathrm{mg} \cdot \mathrm{mL}^{-1}$ of $\mathrm{P}(\mathrm{MMA}-\mathrm{NVP}), \mathrm{P}(\mathrm{MMA}-\mathrm{NVP})-\mathrm{I}, \mathrm{P}(\mathrm{AN}-\mathrm{NVP})$, and $\mathrm{P}(\mathrm{AN}-$ NVP)-I for $30 \mathrm{~min}$. 

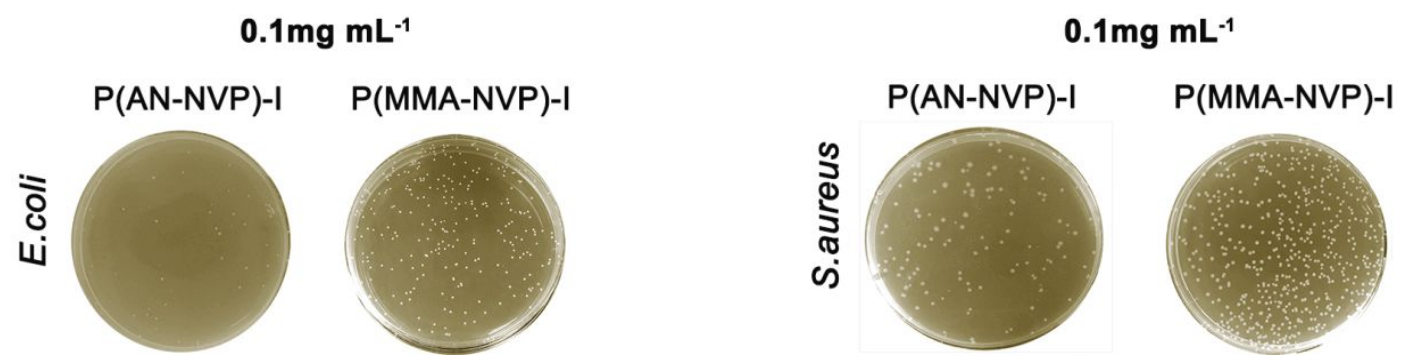

Figure S7. Survival of E. coli and S. aureus after treatment respectively with $0.1 \mathrm{mg} \cdot \mathrm{mL}^{-1}$ of P(AN-NVP)-I and P(MAM-NVP)-I for 30 min. 


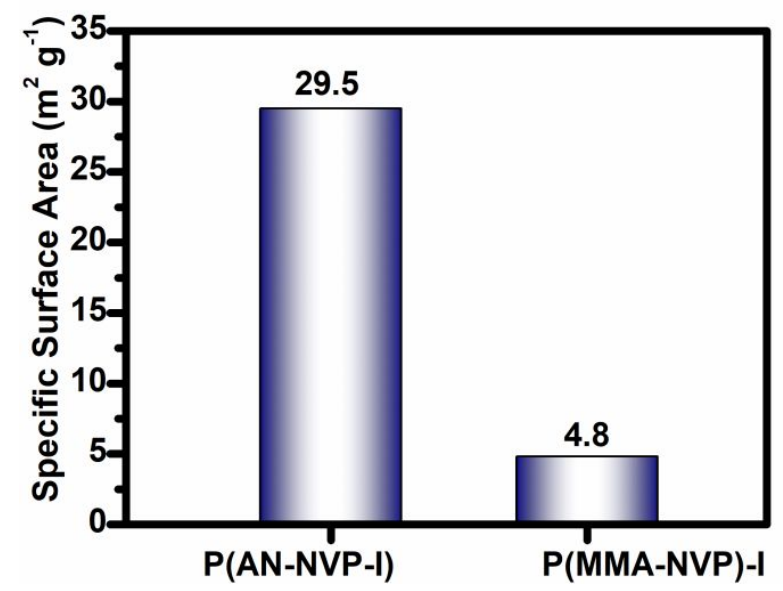

Figure S8. Specific surface area of the P(AN-NVP)-I and P(MMA-NVP)-I. 


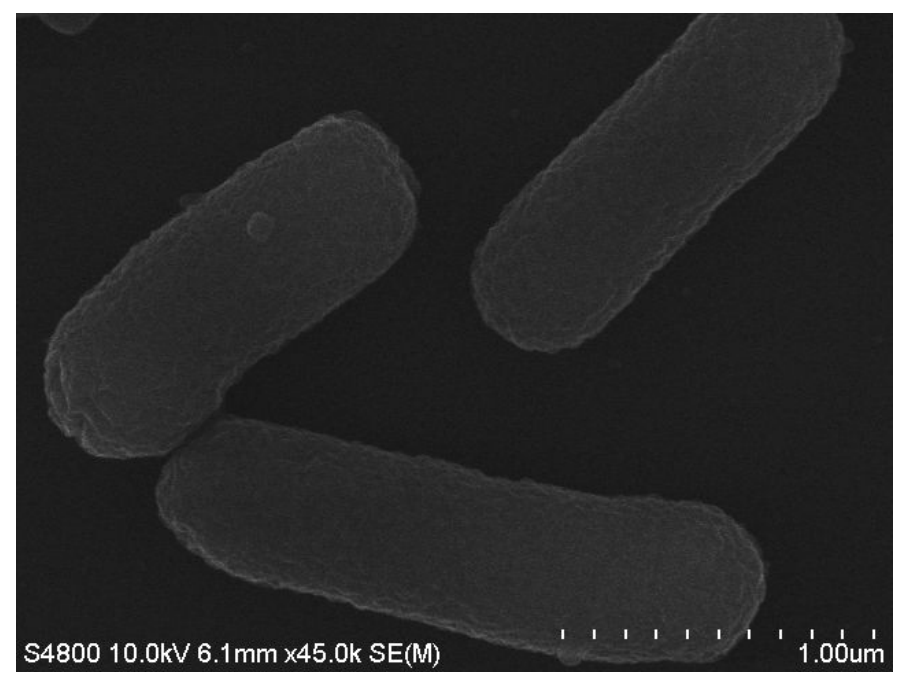

Figure S9. SEM images of E. coli in the absence of samples. 


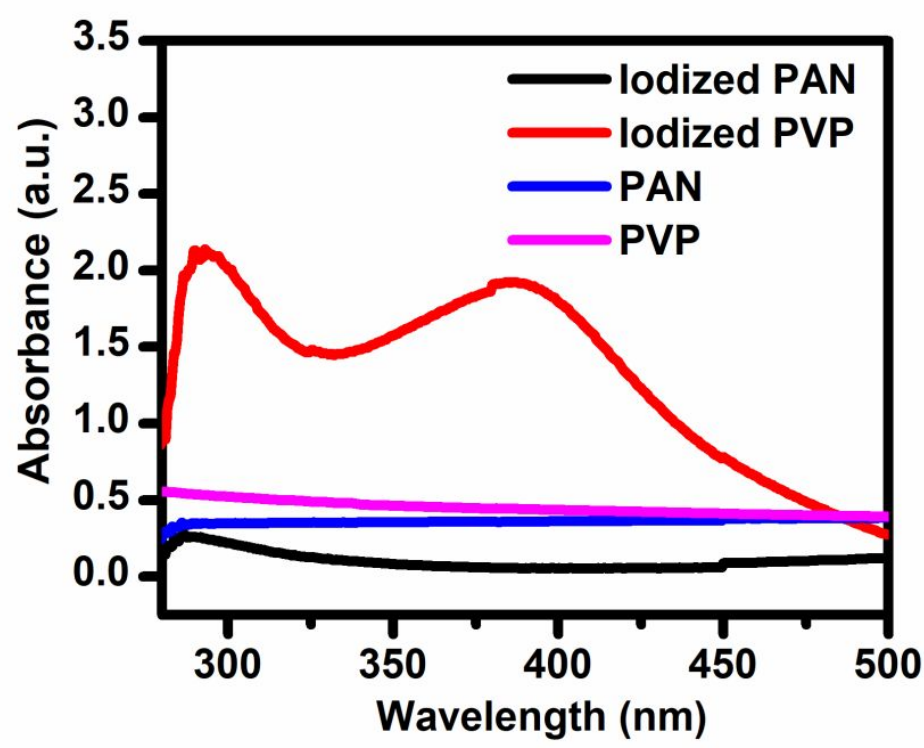

Figure S10. UV-vis spectra of iodized PAN, iodized PVP, PAN and PVP. 